3 Bene Poelsema, Adil Acun, Lisette Schouten, Floor Derkink, Martina Tsvetanova, Zhiguo

4 Zhang, Harold J.W. Zandvliet, Arie van Houselt

5 Physics of Interfaces and Nanomaterials, MESA+ Institute for Nanotechnology,

6 University of Twente, P.O.B. 217, 7500AE Enschede, The Netherlands

\title{
8 Abstract
}

9 The ionic component of the strong bond in hexagonal boron nitride (hBN) has been grossly 10 disregarded in literature. Precisely this quantity is demonstrated to govern the shape of 11 monolayer hBN islands grown at high temperatures. HBN zigzag edges are charged and 12 energetically less favorable than the neutral armchair edges, in contrast to those of the purely 13 covalent graphene. Nucleation of hBN islands occurs exclusively on either the inner or the outer 14 corners of substrate steps. Taking into account the charge at edges of hBN islands offers a 15 powerful framework to understand the nucleation of the islands and their orientation with 16 respect the founding steps, as well as various equilibrium shapes, including prominently a right-

17 angled trapezoid. BN dimers are identified as basic building blocks for hBN. A surprisingly strong 18 interaction between $\mathrm{hBN}$ and the pre-existing steps on the moderately reactive $\operatorname{Ir}\left(\begin{array}{lll}1 & 1 & 1\end{array}\right)$

19 substrate is uncovered. Localized charges are probably relevant for all 2D-materials lacking 20 inversion symmetry.

22 Keywords: hexagonal boron nitride, nucleation and growth, $\mathrm{sp}^{2}$ hybridization, charged edges,

23 equilibrium shapes, Schmoluchowski effect, Low Energy Electron Microscopy 
27 The discovery of the spectacular properties of graphene has revolutionized the interest 28 in two-dimensional (2D) materials ${ }^{1,2}$. This holds in particular for the other group IV 29 allotropes silicene and germanene $e^{3,4,5,6}$ and, on the same tide, transition metal 30 dichalcogenides (TMDs) as $\mathrm{MoS}_{2}{ }^{7,8}$. For applications such as electronic, magnetic and 31 (chemical) sensor devices, it is crucially important that these 2D materials can be 32 decoupled from metallic substrates and from each other ${ }^{9,10,11,12}$. Insulating hexagonal 33 boron nitride ( $\mathrm{hBN}$ ) films $\mathrm{s}^{13}$ are widely believed to provide a viable solution and are

34 frequently referred to as "white graphene". HBN grows in a self-limited fashion as a III-V 35 insulating monomolecular, $\mathrm{sp}^{2}$-hybridized layer on many metal substrates, with a 36 bandgap of about $6 \mathrm{eV}^{14,15} \cdot \operatorname{Ir}(111)$ is a preferred substrate due to the suitable lattice

37 match and a moderately weak binding. The hBN monolayers form moiré structures with 38 a strong tendency to align with the substrate $14,16,17,18,19,20$. In general the structural 39 characteristics of hBN and graphene on $\operatorname{Ir}(111)$ show distinct 40 similarities $21,22,23,24,25,26,27,28,29$.

42 In the euphoria evolved on the promising potential of combining graphene with hBN for 43 innovative applications, the decisive role of the ionic components of the boron-nitrogen 44 bond in the growth of ultrathin hBN layers has passed grossly unnoticed. It leads to 45 polar edges which give rise to a divergent Coulomb contribution to the total edge 46 energy (see Supplementary information section I). Our Low Energy Electron Microscopy

47 (LEEM) data reveals a direct relation between polar binding and the shape of hBN 48 monolayers and provides insight in the nature of the growth precursor. Surprisingly, a 49 strong mutual interaction between the "Van der Waals" film and the substrate plays an 50 additional role in the shape of hBN monolayer islands.

52 In their seminal contribution on $\mathrm{hBN}$ on $\operatorname{Ir}(111)$, Farwick zum Hagen et $a l^{14}$ reported a 53 coincident moiré unit mesh of $(12 \times 12)$ hBN cells on $(11 \times 11)$ Ir substrate cells with two 54 oppositely oriented phases. The moiré unit cell is mainly flat with the BN rings about 
$55 \quad 3.58 \AA$ above the uppermost $\operatorname{Ir}(111)$ layer. The moiré unit cell contains a distinct valley 56 with a minimum height of $B N$ of about $2.07 \AA$ above $\operatorname{Ir}(111)$, which anchors the moiré 57 pattern to the substrate ${ }^{14}$.

59 Figure 1 shows snapshots from a LEEM movie taken during growth of $h B N$ on $\operatorname{Ir}(111)$ at $601150 \mathrm{~K}$ (see Supplementary Information section II). The darkish lines show monoatomic 61 steps, multiple steps or step bunches on clean $\operatorname{Ir}(111)$. The dark areas represent growing 62 hBN islands. In all our experiments they nucleate exclusively at steps and initially they 63 have an isosceles (almost equilateral) triangular shape. This threefold symmetry reveals 64 that the island edges preferentially orient along a specific high symmetry direction of 65 the 2D hBN film. Two oppositely oriented isosceles triangles are distinguished. 66 Oppositely oriented triangular islands may start their evolution from the same step. This 67 implies that nucleation can occur at the inner (lower) side of the step as well as at the 68 outer (upper) side of the step. From the about equal occurrence of the events we 69 conclude that there is little to no energetic difference for both types of islands. This is 70 further corroborated by their similar growth rates.

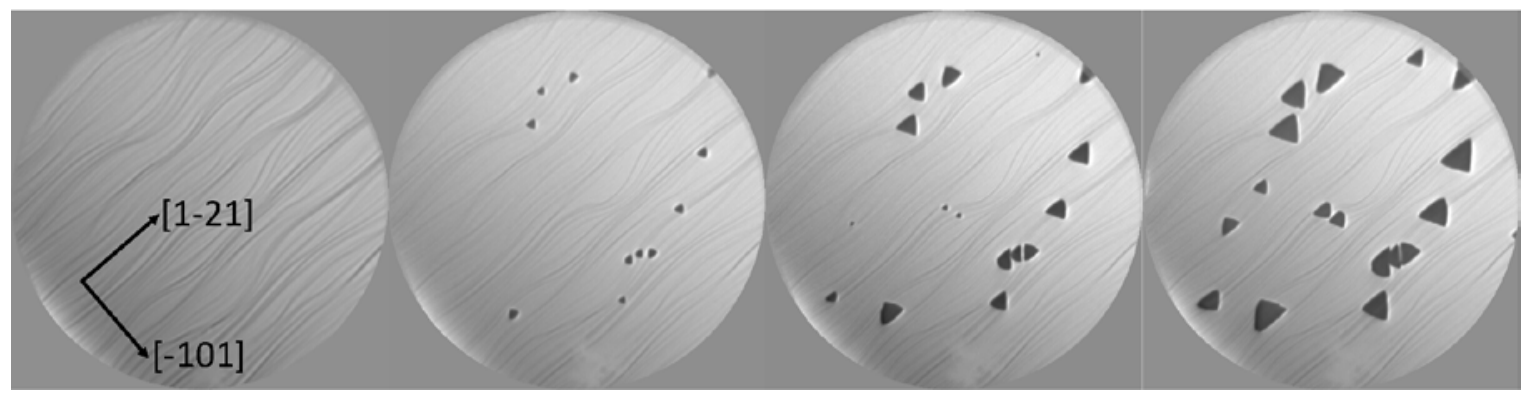

$t=0$ s

$t=120 \mathrm{~s}$

$t=380 \mathrm{~s}$

$t=750 \mathrm{~s}$

Figure 1: Growth of triangular hBN islands.

Snapshots from a LEEM movie taken during growth of hBN (black features) at $1150 \mathrm{~K}$. Field of view is 20 $\mu \mathrm{m}$, electron energy $17 \mathrm{eV}$. Curved lines represent steps which are globally oriented along the [1-21] direction.

The internal structure of $\mathrm{hBN}$ in the oppositely oriented triangles is oppositely oriented as well. Figure 2 shows two selected area diffraction ( $\mu$ LEED) patterns, obtained with an aperture size of $1.4 \mu \mathrm{m}$. The patterns are characteristic of oppositely oriented triangles. 
81 The data, taken at $35 \mathrm{eV}$, shows that the moiré pattern as revealed by the fine structure 82 in the diffraction pattern is nicely aligned to the substrate. The overall patterns are 83 threefold symmetric in both cases, due to the FCC structure of the $\operatorname{Ir}(111)$ substrate. The 84 threefold symmetry is rotated by $180^{\circ}$ for the two patterns. This implies that the 85 opposite orientation of the triangles is accompanied by a $180^{\circ}$ rotated atomic 86 arrangement inside the triangular hBN islands.

\section{Figure 2: Orientation of the oppositely oriented triangles}

Contrast inversed (bright to dark spots) $\mu$ LEED patterns from representatives of both island types. Aperture $1.4 \mu \mathrm{m}$ and electron energy $35 \mathrm{eV}$. The darkish area left from the specular beam is due to secondary electrons, including inelastically scattered ones. The curved lines are an artifact due to digital noise.

The simultaneous presence of both orientations is in line with the vast majority of the literature on the $\mathrm{hBN} / \operatorname{Ir}(111)$ system, following the pioneering paper by Auwärter et al. ${ }^{30}$, where this observation was first made for $\mathrm{hBN}$ on $\mathrm{Ni}(111)$ using a photo-electron diffraction technique. These observations are explained by a preference for the boron atoms to occupy threefold hollow sites on $\operatorname{Ir}(111)$, while the $\mathrm{N}$ atoms prefer on top positions. For one type of flakes the B atoms occupy preferably HCP sites (above a second layer Ir atom) and take FCC positions (above a third layer Ir atom) for the other

104 they are strongly bound. We refer to these types as $\mathrm{H}-\mathrm{hBN}$ and F-hBN, respectively.

105 The threefold symmetric shape of the islands indicates that their edges are of either the 106 zigzag- or the armchair type ${ }^{31}$ (See also Supplementary information section III for a 107 sketch and an estimation for the difference in edge energies).These are oriented along 108 the $<1-10>$ and the $<-1-12>$ azimuth directions of the $\operatorname{Ir}(111)$ substrate, respectively. 
For reasons that become evident below we consider the armchair (along $\langle-1-12>$ ) as the

110 favored edge. This situation is sketched in Fig. 3 for a commensurate hBN structure, 111 while in reality the $\mathrm{hBN}$ is only higher order commensurate with $(12 \times 12) \mathrm{hBN}$ unit cells 112 residing on $(11 \times 11) \operatorname{Ir}(111)$ unit cells. In the valleys of the moiré pattern the $\mathrm{B}$ and $\mathrm{N}$ 113 atoms are chemically

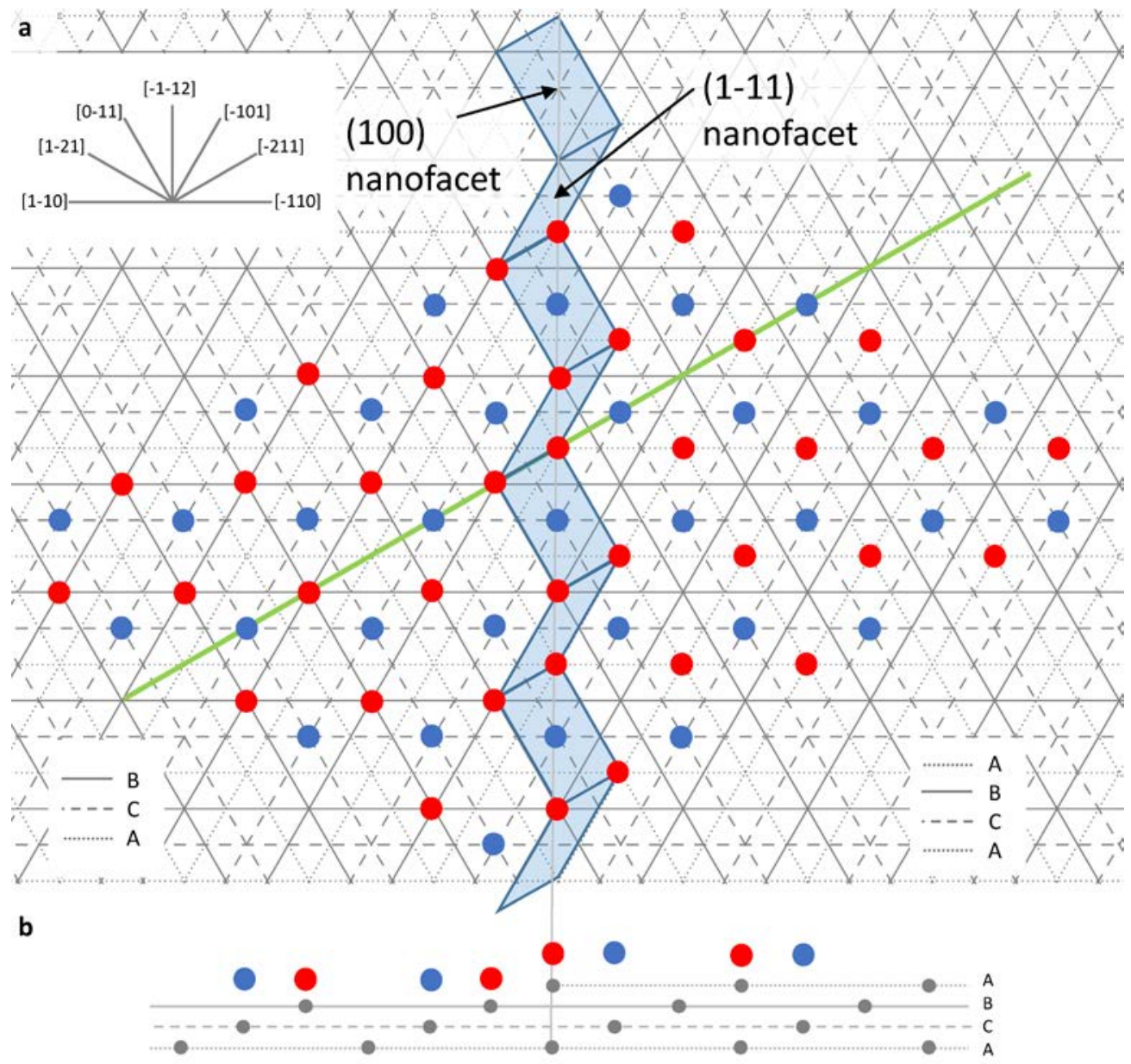

115 Figure 3: Schematic representation of triangular hBN islands.

116 a. Top view of the $\operatorname{Ir}(111)$ substrate with the atomic layer levels indicated by A, B, and C (see inserts). A [117 1-12] oriented atomic step is sketched in the center (azimuth directions are indicated in the upper left 118 insert). The step up direction is from left (stacking order BCABCA) to right (stacking order $A B C A B C$ ). The 119 (1-11) and (100) nanofacets within the step are indicated by the blue rhombi and rectangles, respectively. 120 Triangular $\mathrm{sp}^{2}$ hybridized $\mathrm{hBN}$ islands are shown on the different terraces, with the $\mathrm{B}$ - and $\mathrm{N}$-atoms as blue and red circles, respectively. b. Side view along the [-211] direction (left to right).

bound to the substrate. The $\mathrm{B}$ and $\mathrm{N}$ atoms outside the valleys assume less well-defined positions with respect to the $\operatorname{Ir}(111)$ unit cells and are much more weakly bound to $\operatorname{Ir}^{14}$. 
125 Fig. 3 sketches the situation in the valleys of the moire profile with strong binding 126 (chemisorption). The left and right hand side triangles represent H-hBN and F-hBN, 127 respectively. Note that the size of the valleys is much smaller than the area shown in Fig. 1283.

129 The distinct role of the steps is already clear from the observation that nucleation of $130 \mathrm{hBN}$ islands occurs exclusively on step edges. This holds strictly for the relatively high 131 growth temperatures in the present study. The nucleation occurs at about equal rates 132 on top of steps as well as at the inner corners. These nucleation sites appear to pin the 133 moiré plates and consequently determine whether the hBN islands are of $\mathrm{H}$ - or F-type. 134 This specific nucleation behavior is attributed to the consequences of Smoluchowski 135 smoothing of the electron density contour around atomic steps ${ }^{32}$. This leads to the 136 formation of dipoles around steps with a reduced electron density at the upper part of 137 the step and excess electron density at the inner corners. As a result, the work function 138 of metal surfaces decreases with increasing step density ${ }^{33}$. Electron density smoothing 139 at steps has been demonstrated directly by thermal helium atom scattering, which 140 senses electron density contours ${ }^{34}$. The Smoluchowski effect at steps is generic and is 141 increasingly significant for more open step directions, i.e. it is stronger for <-1-12> steps 142 than for $<1-10>$ steps. It may even result in sizeable inward relaxation of the protruding 143 upper step atoms $\mathrm{s}^{35}$. The decisive role of the steps for the growth of H- or F-type hBN is 144 now understood straightforwardly. An $\mathrm{N}$ atom (red) carries a net negative charge and a $145 \mathrm{~B}$ atom inside $\mathrm{hBN}$ is positively charged ${ }^{36}$. Consequently, the $\mathrm{N}$ atom at the hBN edges is 146 bound most strongly on top of the protruding Ir atom in the upper level with lacking 147 conduction electrons. From there the $\mathrm{B}$ and other $\mathrm{N}$ atoms assume sites governed by 148 the threefold symmetries of $\operatorname{Ir}(111)$ and hBN. As illustrated in Fig. 3, this gives rise to the 149 growth of F-type hBN when nucleation takes place at the upper step edge. In a similar 150 way when nucleation takes place at the lower step edge, the B atom (blue) with net 151 positive charge, takes a position with the highest coordination and excess electron 152 density, which is (close to) the centre of the (1-11) nanofacets inside the step. From 153 there the hBN grows naturally as H-type following the rules imposed by the threefold 
154 symmetry of both $\mathrm{hBN}$ and $\operatorname{Ir}(111)$. This way we find a natural explanation for the 155 anchor sites of the moiré pattern, the type of the resulting hBN and the direction of 156 growth observed experimentally. An attendant argument for the resulting orientation of 157 the flakes is that all edges are of the armchair type. Within one period along the edge 158 the outermost $\mathrm{B}$ and $\mathrm{N}$ atom lack both one binding partner compared to atoms in the 159 interior of the hBN island. As a result they will be charged. Their net charges, however, 160 cancel each other and the total (straight) edge is therefore charge neutral.

161 The morphology and detailed growth behavior of both types of triangles differ during 162 more advanced stages of growth. In order to understand the different propagation of $163 \mathrm{hBN}$ across substrate steps we consider its moiré structure in some more detail. It 164 consists of dominant "flat" Van der Waals parts at a distance of about $3.58 \AA$ above the 165 outermost $\operatorname{Ir}(111)$ layer. It has relatively deep and narrow valleys in which the $B$ and $N$ 166 atoms are chemically bound and locally reside at only about $2.07 \AA$ above Ir. These 167 valleys function as anchor sites and determine the alignment with respect to the $\operatorname{Ir}(111)$ 168 and also whether one deals with H-hBN of F-hBN locally. The distance between two 169 adjacent valleys is about $42 \AA$ along the close packed directions on $\operatorname{Ir}(111)$. Nucleation of $170 \mathrm{hBN}$ takes place on either the outer or the inner corner of a [-1-12] oriented step, which 171 leads to oppositely oriented isosceles triangles; several examples are displayed in Fig. 1.

172 The occurrence of both types is about equal, which applies also for their growth rates. 173 However, the motion of their centre of gravity is different. This is illustrated in Fig. 4.

174 The sketch in 4a shows a triangular island of which the horizontal side is pinned at the 175 lower side of an atomic step and the island grows from high to low. The sketch in $4 \mathrm{~b}$ 176 shows also a triangular island. It nucleated at an atomic step, but this time it is able to 177 grow across a descending step. This behavior agrees with the actually measured 178 situation underneath. A possible minor thermal drift would be identical in both cases. 179 For islands nucleated at the outer corners (right hand side) the first anchoring sites are 180 close to the descending step and the moire surface can simply expand from the step. 181 The Van der Waals part of the profile can easily bend and nestle to the lower terrace 182 before a next valley is formed at a lateral distance of $\sim 42 \AA$. After nucleation in the inner 
corner near an ascending step the islands also grows readily away from the corner.

184 However, crossing the ascending corner is now much more difficult since the Van der

185 Waals part of the profile must be lifted by an additional $2.22 \AA$ being the $\operatorname{Ir}(111)$ step 186 height along a small lateral distance of a few Ångstroms. The required bending is quite 187 severe and is considered unlikely. This reasoning implies that the staircase formed by 188 the step trains in Fig. 4 (and in Fig.1) goes downward in the direction of the arrow (from 189 upper left to lower right).
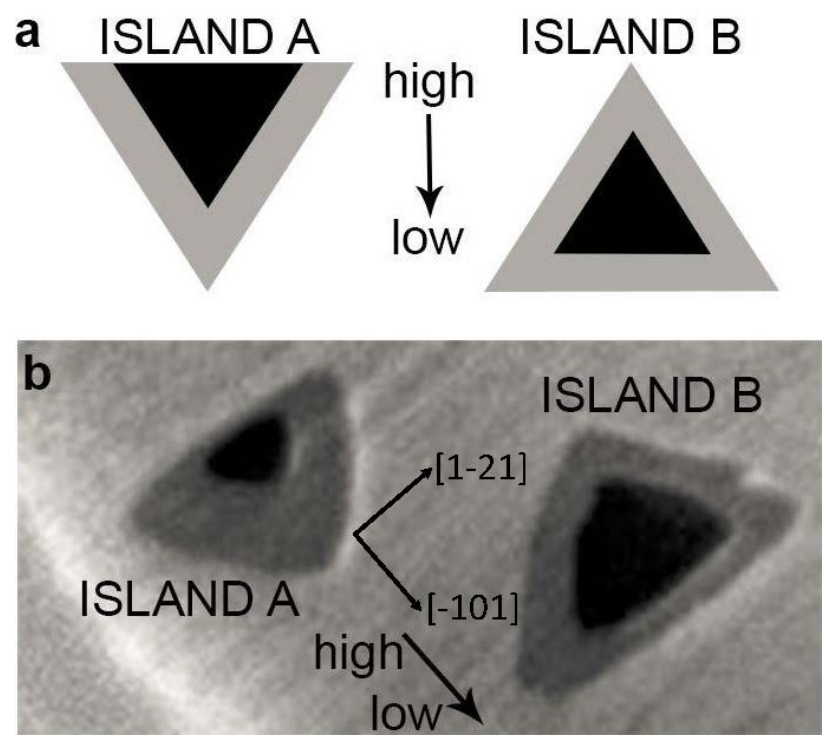

Figure 4: Growth of triangular islands across terraces

HBN islands at early (black) and a more advanced stage of growth (grey). a. Idealized sketch of triangular hBN islands nucleated at an atomic step. b. Corresponding experimental islands taken from a movie during growth. The greyish lines represent pre-existing steps on $\operatorname{lr}(111)$. Field of view $3.2 \times 5.8 \mu \mathrm{m}^{2}$. The azimuth directions are indicated.

191 Figure 5a shows a LEEM image of a clean $\operatorname{Ir}(111)$ sample taken at $1150 \mathrm{~K}$. This picture is, 192 at first sight, a great surprise. Two distinct areas, area I (bottom) and area II (top), are 193 observed on which the slightly curved features, which are attributed to step(bunche)s, 194 as in Fig. 1, are oriented perpendicular to each other. The line separating both areas is 195 strikingly straight. We have carefully looked into the possibility of mozaic structures 196 (microcrystallites) to explain this observation. To this end we compared $\mu$ LEED patterns 197 taken at areas I and II at a broad range of electron energies from about 40 to $200 \mathrm{eV}$. No 198 differences between both sets are observed (cf Figs. 5b and 5c). This way we rule out 
199 the possibility of different local crystal structures to explain the difference between 200 areas I and II. As the only possible result one is left with the predominance of steps in 201 both areas along $90^{\circ}$ different azimuth directions. It is well known that for pristine metal $202 \mathrm{fcc}(111)$ surfaces the atomic steps are preferentially oriented along $<1-10>$ directions.

203 We call these the areas I. The areas II then represent those with dominant <-1-12> 204 steps. We note that these step features cannot cross and therefore a straight line

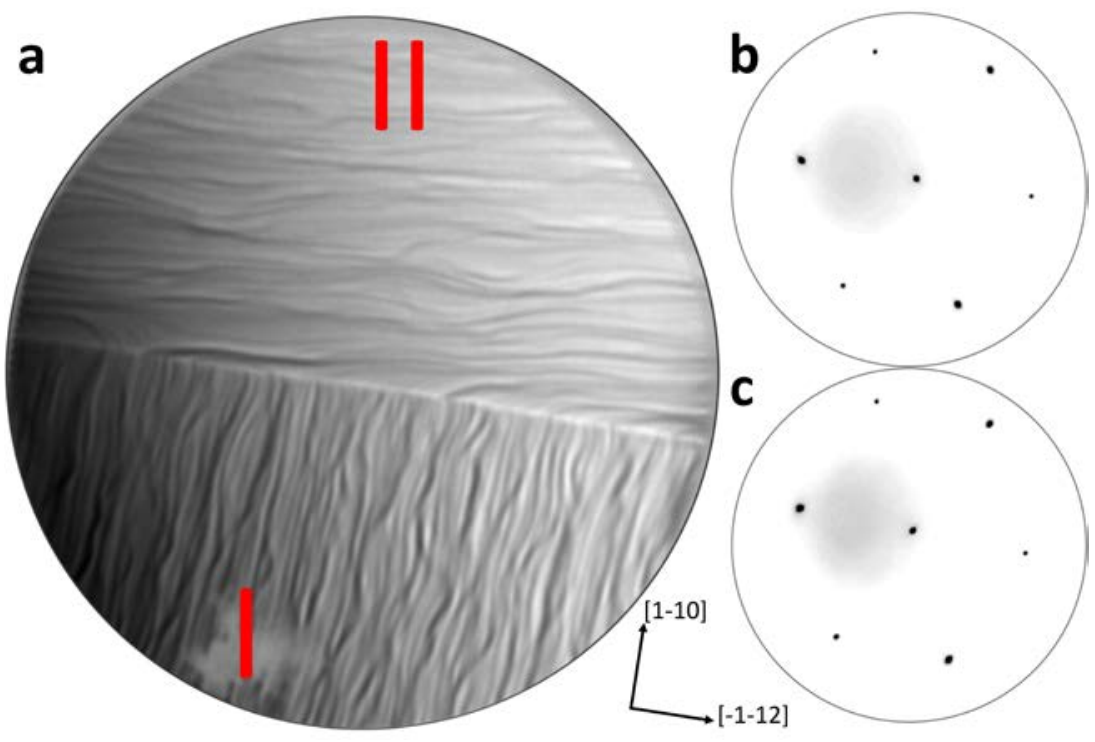

Figure 5: Different preferred step directions after hBN growth

a. LEEM image of clean $\operatorname{Ir}(111)$, FoV $=25 \mu \mathrm{m}$, electron energy $2.5 \mathrm{eV}$. $\mu$ LEED patterns measured with 41.5 $\mathrm{eV}$ electron energy for the upper (b) and lower (c) part of the image in a. The substrate temperature was about $1150 \mathrm{~K}$. The indicated crystallographic directions apply to all panels.

separating both areas fits in this picture. Energetically both step orientations should be quite similar. Steps up and steps down along $<1-10>$ have either $\{111\}$ or $\{100\}$ nanofacets, which are very similar in energy ${ }^{37,38,39}$. The steps along $\langle-1-12>$ also have $\{111\}$ and $\{010\}$ nanofacets (cf. Fig. 3). A strong interaction between $\mathrm{hBN}$ and the steps is held responsible for the observed evolution of the preferred step direction. If that interaction favors the evolution towards $<-1-12>$ oriented steps an increasing integral area II should evolve at the cost of the integral area I. Prolonged hBN growth 
experiments at relatively high temperatures $(900-1200 \mathrm{~K})$ then favor a general rotation of the preferred step orientation away from $<1-10>$ towards $<-1-12>$. In all cases, both areas would remain well separated due to forbidden step crossings. This is exactly what happens after prolonged hBN growth study at high temperatures. We have found an increasing preference for areas II in the course of our prolonged hBN/Ir(111) growth study (See supplementary information section IV for a discussion on the temporal evolution of the change from areas I to II).
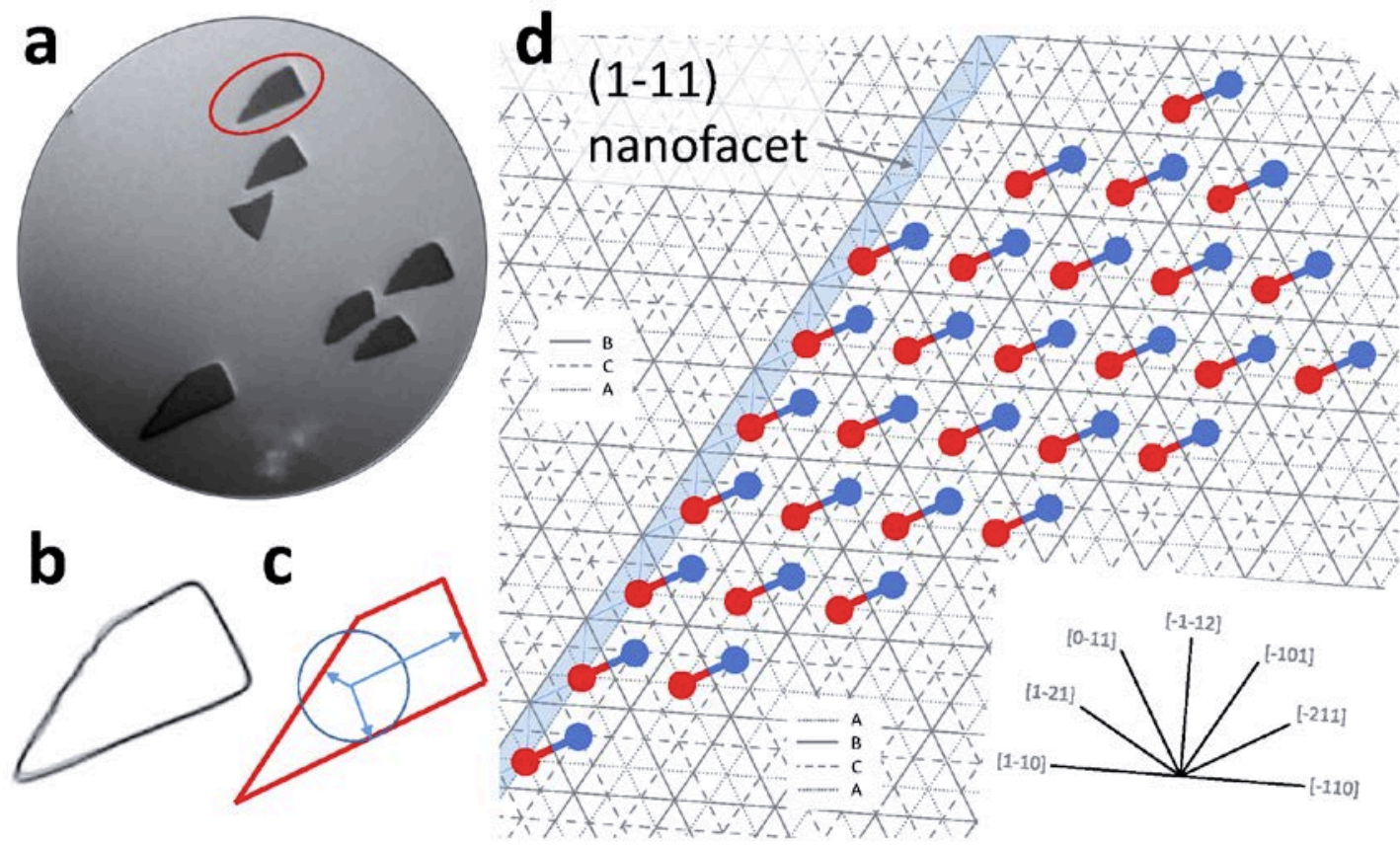

Figure 6: Growth of trapezoidal and triangular hBN islands.

a. Snapshot from a LEEM movie (field of view is $20 \mu \mathrm{m}$, electron energy $16.3 \mathrm{eV}$ ) during growth of hBN on a fresh $\operatorname{Ir}(111)$ sample at $1200 \mathrm{~K}$. b. Normalized contours of 350 subsequent images of the right-angled trapezoidal hBN islands highlighted by the red ellipse in a) The sharp lower left angle is $30^{\circ}$. The left edge nucleated at the [-101] step of the $\operatorname{Ir}(111)$ surface. The areas vary between 0.9 and $4.1 \mu \mathrm{m}^{2}$. c. Sketch of the corresponding Wulff plot (red lines). d. Schematic representation of the right-angled trapezium highlighted by the red ellipse in a. The island is of H-hBN type. The (1-11) nanofacet is indicated by the blue stripe. A similar sketch is possible for $\{100\}$ nanofacets. The constituting $B$ (blue) $-N$ (red) dimers are indicated. 
207 The local preference for steps along $<1-10>$ (area I) or along $<-1-12>$ (area II) leads to 208 pronounced differences in the relative abundance of type $\mathrm{H}$ - or type F-hBN. Figure 1 209 shows snapshots taken from a LEEM movie during the growth of hBN on an area of type 210 II. Figure 6a shows a characteristic snapshot of a LEEM movie (see Supplementary 211 Information section V) taken during initial hBN growth on a fresh $\operatorname{Ir}(111)$ substrate. The 212 predominant step orientation is therefore along $\langle 1-10\rangle$ directions ${ }^{37}$ and the host area is 213 of type I. In contrast to the situation in Fig. 1, triangular islands form a distinct minority. 214 The most abundant hBN islands, nucleated on the [-101] steps in Fig 6a, do not have a 215 triangular shape, but rather exhibit a trapezoidal shape. During the initial stages of the 216 growth, where the mutual influence on and by neighboring islands is still small, these 217 islands have a right-angled trapezoidal shape (see Fig 6a). In the extreme case, they are 218 characterized by sides which make angles of $30^{\circ}$ and $90^{\circ}$ with the longest one of the two 219 parallel sides. This particular shape is unveiled as the equilibrium shape for islands 220 nucleated at straight [-101] step segments with (1-11) nanofacets. This is confirmed by 221 the data gathered in Fig. 6b for a large number (350) of successive images of the right222 angled trapezoidal hBN island highlighted by the red ellipse in Fig. 6a. Fig. 6b shows the 223 normalized outer contours of the island for varying areas from about 0.9 to about 4.1 $224 \mu \mathrm{m}^{2}$. Indeed the shape is identical and does not depend on the size of island. A similar 225 analysis for islands nucleated at different $<-101>$ steps leads to identical results. Minor 226 differences on the left- and right-hand edges are expected and observed due to the 227 strong inherent dependence on the local shape of the founding <-101> steps. We note 228 that considerable deviations from the equilibrium shape occur for larger islands. 229 Depending on whether mass transport occurs via edge diffusion or via 2D surface 230 diffusion the involved times required for establishing equilibration shapes scale with a 231 power law, i.e. as $\mathrm{A}^{4}$ or $\mathrm{A}^{2}$, for an island of size $\mathrm{A}$, respectively $\mathrm{y}^{40}$. The time constant in the 232 experiment is fixed and given by the rate of incidence of the borazine molecules, their 233 decomposition rate and the incorporation rate of the borazine fragments (BN-dimers). 234 Consequently, beyond a given size the islands can no longer maintain their equilibrium 235 shape during progressing growth stages. Departure from equilibrium will also occur 
236 through direct or indirect interactions (shadowing) with neighboring islands. Therefore, 237 the discussion below focuses on initial stages of growth.

238 The equilibrium shape of $\mathrm{hBN}$ islands nucleated at pre-existing [10-1] on type I regions 239 of $\operatorname{tr}(111)$ is now completely defined. The edge of the islands at the parent step is of the 240 zigzag type. The edges pointing away from the ascending step make an angle of $30^{\circ}$ with 241 the step and align along the [-211] azimuth and are thus of the armchair type. The 242 remaining edge exhibits a right angle to [-211], i.e. is aligned along [0-11] (see Fig. 6d). 243 Note that this edge is not of zigzag type, but rather boron terminated. This 244 experimental fact allows important conclusions on the elementary building blocks for 245 the hBN islands. With increasing temperature first dehydrogenation of the borazine 246 molecules takes place. On most metals, in particular transition state metals, the 247 resulting $\mathrm{H}$-atoms desorb associatively. The other extreme at very high temperatures is 248 a complete decomposition as the borazine molecules fall apart in B and $\mathrm{N}$ atoms. In that 249 case the nitrogen atoms also desorb associatively, leaving B behind. Such is indeed the 250 case for $\mathrm{hBN}$ at higher temperatures than currently considered situation ${ }^{21}$. This situation 251 impairs the balance between $\mathrm{N}$ and $\mathrm{B}$ atoms required to grow $\mathrm{hBN}$ and must be avoided.

252 As a result $\mathrm{hBN}$ grows from well defined fragments as either dehydrogenated $(\mathrm{BN})_{3}$ rings 253 or BN dimers. The latter is particularly stable due to the combination of covalent 254 bonding and ionic bonding. It is impossible to arrive at a hBN-island with the obtained 255 equilibrium shape by successive incorporation of intact $(B N)_{3}$ rings. The successive 256 addition of aligned BN dimers is the only option to grow the observed equilibrium 257 shape, as illustrated in Fig. 6b. We therefore conclude that BN-dimers constitute the 258 basic building blocks for the growth of $h B N$ on $\operatorname{Ir}(111)$ at $1200 \mathrm{~K}$.

259 The zigzag edge at the parent [-101] step is charged as the terminating $\mathrm{N}$ atoms lack 260 each one $\mathrm{B}$ nearest neighbor when compared to an $\mathrm{N}$ atom in the centre of the island. 261 The built-in charge along the [-101] step is compensated at the opposite [0-11] edge, 262 which is a natural consequence when the hBN islands are built from BN dimers. The 263 terminating [0-11] edge consists of $B N$ dimers of which each $B$ atom lacks two $N$ nearest 
264 neighbors. The positive charge density of the [0-11] edge is therefore twice as high as 265 that of the negative [-101] edge along the Ir [-101] step and charge neutrality is 266 maintained. We emphasize that the armchair edges [-1-12] and [1-21] are missing in the 267 shape of the right-angled trapezium. A close inspection of Fig. $6 \mathrm{~d}$ reveals that these 268 edges cannot be constructed from BN dimers after nucleation of the island at the [-101] 269 step. Therefore, these missing “inexpensive" edges provide additional evidence that BN270 dimers act as the basic building blocks of the hBN island. Figure 6c shows a sketch of the 271 Wulff plot for the equilibrium shaped island in Fig. 6b. The lowest edge energy is along 272 the founding [-101] step, a relatively low edge energy is realized along [-211], while an 273 energetically unfavourable B termination is achieved along [0-11] by a row of BN dimers.

274 The right angled trapezium shape establishes an extreme. For a slightly curved parent 275 step the edge of the hBN island is composed of a combination of zigzag and armchair 276 segments. Therefore, the opposite edge of the island must be composed of 277 corresponding segments in order to warrant charge neutrality. Consequently, local 278 curvature of the parent steps has a direct impact on the island's shape. As mentioned 279 further above the charge neutral armchair edges are energetically preferred. The fact 280 that the island side away from the parent step is not terminated by "cheap" armchair 281 elements is indicative of the enormous influence of Coulomb induced shape effects.

282 As noted earlier triangular hBN islands are only occasionally observed during growth in 283 type I areas too. These islands also nucleate at a parent [-101] atomic step. Notably this 284 step forms a bisector of the growing isosceles triangle (see Fig. 6a for an illustration). 285 The edges of the isosceles island are again along $<11-2>$ Ir azimuths and are thus of the 286 favorable, energetically cheap, armchair type. The hBN structure inside the triangular 287 islands is rotated by $180^{\circ}$ (or $30^{\circ} \pm \mathrm{n} \cdot 120^{\circ}$ ) compared to the predominant trapezoids. 288 Attempts to construct isosceles triangular F-hBN islands with a bisector along a <-101> 289 and armchair edges, however, fail. They all lead to a non-negligible charging at the 290 bisector. It builds up linearly with the growing island size. We suggest that nature does 291 better and propose a model for the triangular islands, shown in Fig. 7. The parent [-101] 
step forming the bisector, is indicated with blue rectangles and has (1-11) nanofacets.

293 The first BN-dimer row is oriented perpendicular to the step. As such they are the 294 complement of the situation of the right-angled trapezium (see Fig. 6d) where the BNdimers in the first row are oriented at $30^{\circ}$ from the step. All edges are of the armchair type and the total triangular island is built up from BN dimers as building blocks. It is

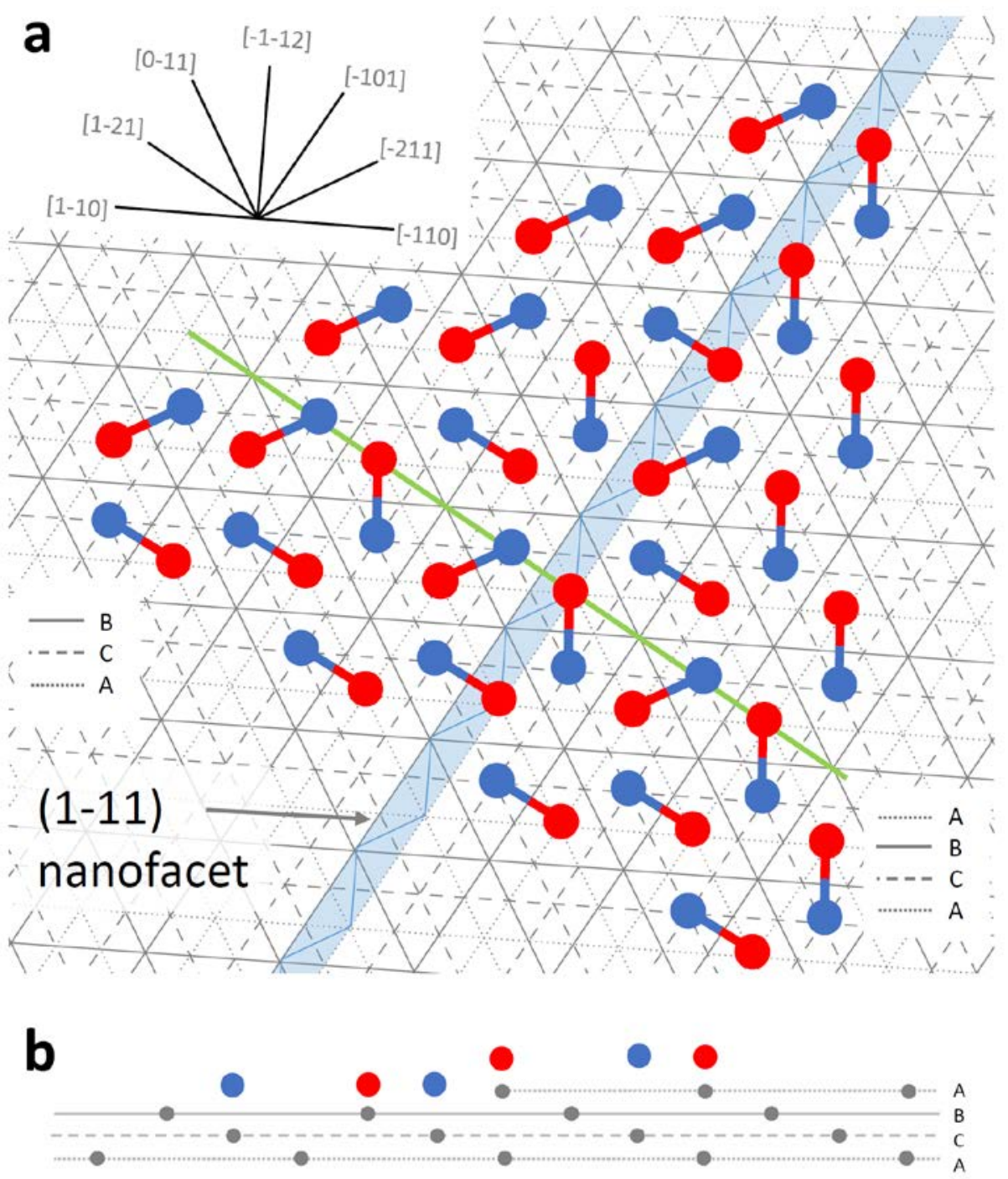

Figure 7: Schematic representation of triangular $h B N$ islands with $\langle 110\rangle$ steps as bisector

a. Schematic representation of the triangular hBN islands in Fig 4a. The [-101] step edge with a (1-11) nanofacet is shown by the blue rectangles. A similar sketch is possible for $\{010\}$ nanofacets. The edges are of the armchair type. Step up from left to right. Left: $\mathrm{H}-\mathrm{hBN}$, right: F-hBN. The constituting $\mathrm{B}(\mathrm{blue})-\mathrm{N}$ (red) dimers are indicated. $\mathbf{b}$. Side view along the [1-21] direction (green line in a). 
easily seen that the total construction is charge neutral and thus no Coulomb based contribution to the total energy of the island is present, including the region around the

301 bisector. Further growth will maintain the energetically favored armchair edges. The left

302 half of the triangle (on the lower terrace) is of H-hBN type, while the right half (on the

303 higher terrace) is of F-hBN type. Indications for different types of hBN within one 304 triangle have indeed been observed ${ }^{41}$. However, we have not observed a clear 305 indication for hybrid hBN inside one island. It is easy to conceive that the structure is 306 anchored or pinned by the situation at the upper step edge (the $\mathrm{N}$ atoms would prefer 307 the sites on top of the step due to Smoluchowski electron density smoothening. 308 Maintaining this anchoring the hBN blanket may well be continued in the F-hBN mode.

309 Triangular hBN islands have been reported by many authors (e.g. ${ }^{41,14}$ ). Following 310 Auwärter et $a l .{ }^{30}$, these authors arrived at the conclusion that these triangles lead to 311 islands terminated by either B- or N-rich edges. That is correct indeed for ideal islands 312 on ideal (stepless) terraces, which require unbalanced $\mathrm{B}$ and $\mathrm{N}$ atom numbers and thus 313 total decomposition. However, our current findings show exclusive nucleation at steps.

314 Combined with BN-dimers as constituting entities, this provides a natural way to break 315 the three-fold symmetry condition and ensures balanced quantities of $\mathrm{B}$ and $\mathrm{N}$ in the 316 hBN island (edges). Our results are thus inconsistent with an exclusive B termination of $317 \mathrm{hBN}$ island edges ${ }^{14}$.

\section{Conclusions}

320 We demonstrate the crucial role of ionic binding aspects in $\mathrm{hBN}$ on the binding to $321 \operatorname{Ir}(111)$, the orientations and locations of the islands and their equilibrium shapes. A 322 direct consequence of the ionic bonds is that zigzag edges are charged, while armchair 323 edges remain neutral. A careful consideration of Coulomb interactions, in combination 324 with Smoluchowski smearing of the electron density at step edges leads to a consistent 325 picture of $\mathrm{hBN}$ on $\operatorname{Ir}(111)$. Since these aspects (ionic binding and electron density 326 smearing at steps) are generic we suggest that this picture guides more generally the 
327 understanding of hBN growth on (quite) strongly interacting metal substrates.

328 Moreover, BN dimers are identified as the basic building blocks of the hBN islands.

329

330 Methods

331

332 An Elmitec LEEM III with a base pressure below $1 \times 10^{-10} \mathrm{mbar}$ was used to study the 333 growth of hBN on $\operatorname{Ir}(111)$. Ir(111) single crystals (Surface Preparation Laboratory) were 334 cleaned by subsequent alternating cycles of Argon ion sputtering and annealing in 335 oxygen environment at $1300 \mathrm{~K}$, with subsequent flash annealing at $1600 \mathrm{~K}$ before each 336 measurement. HBN was removed by annealing at $1300 \mathrm{~K}$ and subsequently the sample 337 was cleaned as described above. No traces of contamination were observed using Auger 338 Electron Spectroscopy. Borazine was purchased from Chemos GmbH.

\section{Acknowledgement}

342 We thank the Nederlandse organisatie voor wetenschappelijk onderzoek (NWO) for 343 financial support. 


\section{References}

[1] Novoselov, K. S., Geim, A. K., Morozov, S. V., Jiang, D., Zhand, Y., Dubonos, S. V., Grigorieva, I. V. \& Firsov, A. A. Electric field effect in atomically thin carbon films. Science 306, 666-669 (2004).

[2] Geim, A. K. \& Novoselov, K. S. The rise of graphene. Nat. Mater. 6, 183-191 (2007).

[3] Vogt, P., De Padova, P., Quaresima, C., Avila, J., Frantzeskakis, E., Asensio, M. C., Resta, A., Ealet, B., \& Le Lay, G. Silicene: compelling experimental evidence for graphenelike twodimensional Silicon. Phys. Rev. Lett. 108, 155501 (2012).

[4] Cahangirov, S., Topsakal, M., Aktürk, E., Sahin, H., \& Ciraci, S. Two- and one-dimensional honeycomb structures of silicon and germanium. Phys. Rev. Lett. 102, 236804 (2009).

[5] Bampoulis, P., Zhang, L., Safaei, A., van Gastel, R., Poelsema, B., \& Zandvliet, H.J.W. Germanene termination of $\mathrm{Ge}_{2} \mathrm{Pt}$ crystals on $\mathrm{Ge}(110)$. J. Phys. Condens. Matter 26, 442001 (2014).

[6] Zhang, L., Bampoulis, P., Rudenko, A.N., Yao, Q., van Houselt, A., Poelsema, B. \& Zandvliet, H.J.W. Structural and electronic properties of germanene on $\mathrm{MoS}_{2}$. Physical Review Letters 116, 256804 (2016).

[7] Mak, K.F., Lee, C., Hone, J., Shan, J., \& Heinz, T.F. Atomically thin MoS2: a new direct-gap semiconductor. Phys. Rev. Lett. 105, 136805 (2010).

[8] Li, H., Zhang, Q., Yap, C.C.R., Tay, B.K., Edwin, T.H.T., Olivier, A. \& Baillargeat, D. From bulk to monolayer $\mathrm{MoS}_{2}$ : Evolution of Raman scattering. Adv. Funct. Mater. 22, 1385-1390 (2012).

[9] Xu, M., Liang, T., Shi, M.\& Chen, H. Graphene like two-dimensional materials. Chem. Rev. 113, 3766-3798 (2013).

[10] Schulz, F., Drost, R., Hämäläinen, S. K. \& Liljeroth, P. Templated self-assembly and local doping of molecules on epitaxial boron nitride. ACS Nano 7, 11121-11128 (2013).

[11] Gehring, P, Gao, B.F., Burghard, M. \& Kern, K. Growth of high-mobility $\mathrm{Bi}_{2} \mathrm{Te}_{2} \mathrm{Se}$ nanoplatelets on hBN sheets by van der Waals epitaxy. Nano Lett. 12, 5137-5142 (2012).

[12] Dean, C.R., Young, A.F., Meric, I., Lee, C., Wang, L., Sorgenfrei, S., Watanabe, K., Taniguchi, T., Kim, P., Shepard, K. L. \& Hone, J. Boron nitride substrates for high-quality graphene electronics. Nat. Nanotechnol. 5, 722-726 (2010).

[13] Corso, M., Auwärter, W., Muntwiler, M., Tamai, A., Greber, T., \& Osterwalder, J. Boron nitride nanomesh. Science 303, 217-220 (2004).

[14] Farwick zum Hagen, F.H., Zimmermann, D.M., Silva, C.C., Schlueter, C., Atodiresei, M., Jolie, W., Martínez-Galera, A.J., Dombrowski, D., Schröder, U.A., Will, M., Lazić, P., Caciuc, V., Blügel, S., Lee, T-L., Michely, T. \& Busse, C. Structure and growth of hexagonal boron nitride on $\operatorname{Ir}(111)$. ACS Nano 10, 11012-11026 (2016).

[15] Cassabois, G., Valvin, P. \& Gil, B., Hexagonal boron nitride is an indirect bandgap semiconductor. Nature Photonics 10, 262-266 (2016).

[16] Dong, G., Fourré, E.B., Tabak, F.C. \& Frenken, J.W.M. How boron nitride forms a regular nanomesh on Rh(111). Phys. Rev. Lett. 104, 096102 (2010). 
[17] Orlando, F., Lacovig, P., Omiciuolo, L., Apostol, N.G., Larciprete, R., Baraldi, A. \& Lizzit, S. Epitaxial growth of a single-domain hexagonal boron nitride monolayer. ACS Nano 8, 1206312070 (2014).

[18] Orlando, F., Larciprete, R., Lacovig, P., Boscarato, I., Baraldi, A. \& Lizzit, S. Epitaxial growth of hexagonal boron nitride on Ir (111). J. Phys. Chem. C 116, 157-164 (2012).

[19] Usachov, D., Fedorov, A., Vilkov, O., Adamchuk, V. K., Yashina, L. V., Bondarenko, L., Saranin, A. A., Grüneis, A. \& Vyalikh, D. V. Experimental and computational insight into the properties of the lattice-mismatched structures: Monolayers of h-BN and graphene on $\operatorname{Ir}(111)$. Phys. Rev. B 86, 155151 (2012).

[20] Schulz, F., Drost, R., Hämäläinen, S.K., Demonchaux, T., Seitsonen, A. P. \& Liljeroth, P. Epitaxial hexagonal boron nitride on $\operatorname{Ir}(111)$ : A work function template. Phys. Rev. B 89, 235429 (2014).

[21] Petrović, M., Hagemann, U., Horn-von Hoegen, M. \& Meyer zu Heringdorf, F.-J. Microanalysis of single-layer hexagonal boron nitride islands on $\operatorname{Ir}(111)$. Appl. Surf. Sci. 420, 504510 (2017).

[22] van Gastel, R., N'Diaye, A. T., Wall, D., Coraux, J., Busse, C., Buckanie, N. M., Meyer-zuHeringdorf, F., Horn von Hoegen, M., Michely, T. \& Poelsema, B. Selecting a single orientation for millimeter sized graphene sheets. Appl. Phys. Lett. 95, 121906 (2009).

[23] N'Diaye, A. T., van Gastel, R., Martínez-Galera, A. J., Coraux, J., Hattab, H., Wall, D., Meyerzu-Heringdorf, F., Horn von Hoegen, M., Gómez-Rodríguez, J. M., Poelsema, B., Busse, C. \& Michely, T. In situ observation of stress relaxation in graphene. New J. of Phys. 11, 113056 (2009).

[24] Sutter, P., Sadowski, J. T. \& Sutter, E. Graphene on Pt(111): Growth and substrate interaction. Phys. Rev. B 80, 245411 (2009).

[25] Sutter, P. \& Sutter, E. Microscopy of Graphene Growth, Processing, and Properties. Adv. Funct. Mat. 23, 2617-2634 (2013) and references therein.

[26] Loginova, E., Bartelt, N. C., Feibelman, P. J. \& McCarty, K. F. Evidence for graphene growth by C cluster attachment. New J. Phys. 10, 093026 (2008).

[27] Loginova, E., Nie, S., Thürmer, K. Bartelt, N. C. \& McCarty, K. F. Defects of graphene on $\operatorname{Ir}(111)$ : Rotational domains and ridges. Phys. Rev. B 80, 085430 (2009).

[28] Rogge, P. C., Thürmer, K., Foster, M. E., McCarty, K. F., Dubon, O. D. \& Bartelt, N. C. Realtime observation of epitaxial graphene domain reorientation. Nature Comm. 6, 6880 (2015).

[29] Rogge, P. C., Nie, S., McCarty, K. F., Bartelt, N. C. \& Dubon, O. D. Orientation-dependent growth mechanisms of graphene islands on $\operatorname{Ir(111).~Nano~Lett.~15,~170-175~(2015).~}$

[30] W. Auwärter, M. Muntwiler, J. Osterwalder \& T. Greber, Defect lines and two-domain structure of hexagonal boron nitride films on Ni(111). Surf. Sci. 545, L735 (2003).

[31] K. Nakada, M. Fujita, G. Dresselhaus, M.S. Dresselhaus, Edge state in graphene ribbons: Nanometer size effect and edge shape dependence. Phys. Rev. B 54, 17954 (1996).

[32] Smoluchowski, R. Anisotropy of the electronic work function of metals. Phys. Rev. 60, 661674 (1941). 
[33] Poelsema, B., Palmer, R. L. \& Comsa, G. Helium scattering and work function investigation of CO adsorption on Pt(111) and vicinal surfaces. Surf. Sci. 123, 152-164 (1982). [34] Bedrossian, B., Poelsema, B., Rosenfeld, G., Jorritsma, L. C., Lipkin, N. N. \& Comsa, G. Electron density contour smoothening for epitaxial Ag islands on $\mathrm{Ag}(100)$. Surf. Sci. 334, 1-9

463 (1995).

[35] Visscher, B., Boers, A. L., Verheij, L. K. \& Poelsema, B. Relaxation of the edge atoms of a stepped Cu(410) surface. Appl. Surf. Sci. 26, 121-128 (1986).

[36] Grad, G. B., Blaha, P., Schwarz, K., Auwärter, W. \& Greber, T. Density functional theory investigation of the geometric and spintronic structure of $h-\mathrm{BN} / \mathrm{Ni}(111)$ in view of photoemission and STM experiments. Phys. Rev. B 68, 085404 (2003).

Greber, T. Graphene and Boron Nitride single layers, arxiv:0904.1520 (2009).

[37] Bott, M., Hohage, M., Michely, T \& Comsa, G. Pt(111) reconstruction induced by enhanced Pt gas-phase chemical potential. Phys. Rev. Lett. 70, 1489-1492 (1993).

[38] Jacobsen, J., Jacobsen, K. W. \& Nørskov, J.K. Island shapes in homoepitaxial growth of Pt(111). Surf. Sci. 359, 37-44 (1996).

[39] Rost, M. J., Michely, T. \& Comsa, G. Comment on "Self-diffusion and dynamic behavior of atoms at step edges on iridium surfaces. Phys. Rev. B 57, 1992-1994 (1998).

[40] Morgenstern, K., Rosenfeld, G., Poelsema, B. \& Comsa G. Brownian motion of vacancy islands on Ag(111). Phys. Rev. Lett. 74, 2058-2061 (1995). 